\title{
French translation and validation de Liege of the Exercise-Induced Leg Pain Questionnaire
}

\section{SpORt?}

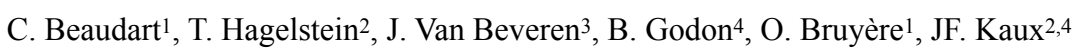

1. Department of Public Health, Epidemiology and Health Economics, University of Liège, Liège, Belgium.

2. Physical Medicine and Sports Traumatology Department, University and University Hospital of Liège, Liège, Belgium.

\section{Introduction}

The "Exercise-Induced Leg Pain " (EILP) questionnaire has been developed to evaluate the severity of symptoms and sports ability in individuals with exercise-induced leg pain. Purpose: We aimed to translate and cross-culturally adapt this questionnaire into French and to study the reliability and validity of the French-translated version (EILP-F).

\section{Material and methods}

Translation and cross-culturally adaptation of the original EILP (EILP-G) was performed according to established guidelines. The translation part was articulated in six stages as recommended by Beaton et al. To validate the EILP-F questionnaire, 84 subjects were recruited ( 28 pathological patients with a confirmed diagnosis of chronic leg pain, 28 asymptomatic sport students, 28 athletes healthy control). Discriminative power of the questionnaire was tested as well as reliability (internal consistency, test-retest reliability after a 7-10-day interval), construct validity and floor/ ceiling effects.

\section{Results}

the EILP-F version of the questionnaire has been generated without any major difficulties. The ability of the questionnaire to discriminate the three groups of subjects has been showed with a total score of $61.0 \pm 18.5$ for the pathologic group; $93.9 \pm 7.57$ for the asymptomatic group and $94.1 \pm$ 9.79 for the control group ( $p$-value adjusted on age $=0.008$ ). A high internal consistency (Cronbach's alpha of 0.93) and an excellent test-retest radiality (ICC of 0.98 (95\% Cl 0.97-0.99, $p<0.001)$ ) indicated that the EILP-F is reliable. The EILP-F also demonstrated a good construct validity against different subscales of the Short Form-36 questionnaire, a generic quality of life questionnaire, with more than $87 \%$ of prespecified hypotheses confirmed. Finally, no floor or ceiling effects were observed.
Questionnaire pour déterminer l'importance des douleurs chroniques à la jambe occasionnées par le sport

Chère patiente / cher patient,

Veuillez s'il vous plaît cocher la case qui convient le mieux pour décrire votre état durant la dernière semaine. En cas de symptômes bilatéraux, répondez en fonction du côté le plus douloureux. Si l'activité spécifiée est limitée par d'autres facteurs que des plaintes à la jambe, veuillez cocher «non applicable ».

Suite à vos douleurs à la jambe, vous éprouvez.

\begin{tabular}{|c|c|c|c|c|c|c|}
\hline & $\begin{array}{l}\text { Aucune } \\
\text { difficulté }\end{array}$ & $\begin{array}{l}\text { Difficulté } \\
\text { minime }\end{array}$ & $\begin{array}{l}\text { Difficulté } \\
\text { modérée }\end{array}$ & $\begin{array}{l}\text { Difficulté } \\
\text { extrême }\end{array}$ & $\begin{array}{c}\text { Difficulté rendant } \\
\text { l'activité impraticable }\end{array}$ & $\begin{array}{c}\text { Non } \\
\text { applicable }\end{array}$ \\
\hline \multicolumn{7}{|l|}{$\begin{array}{l}\text { Lors du démarrage de la course } \\
\text { (courir, faire du jogging) }\end{array}$} \\
\hline \multicolumn{7}{|l|}{$\begin{array}{l}\text { Après environ } 10 \\
\text { minutes de course }\end{array}$} \\
\hline \multicolumn{7}{|l|}{$\begin{array}{l}\text { Après environ } 15 \\
\text { minutes de course }\end{array}$} \\
\hline \multicolumn{7}{|l|}{$\begin{array}{l}\text { Après } 30 \text { minutes } \\
\text { de course ou plus }\end{array}$} \\
\hline \multicolumn{7}{|l|}{ A l'impulsion d'un saut } \\
\hline \multicolumn{7}{|l|}{ A la réception d'un saut } \\
\hline \multicolumn{7}{|l|}{$\begin{array}{l}\text { Lors de démarrages } \\
\text { ou d'arrêts rapides }\end{array}$} \\
\hline \multicolumn{7}{|l|}{$\begin{array}{l}\text { Lors de changements de direction } \\
\text { ou de mouvements latéraux }\end{array}$} \\
\hline \multicolumn{7}{|l|}{$\begin{array}{l}\text { Lors d'activités peu sollicitantes } \\
\text { (marcher) }\end{array}$} \\
\hline $\begin{array}{l}\text { Pour exercer une activité } \\
\text { sportive sans limite }\end{array}$ & & & & & & \\
\hline
\end{tabular}

\section{Conclusion}

The EILP-F questionnaire is consistent, valid and reliable for evaluating the French-speaking patients with chronic exercise-induced leg pain. 